\title{
Ultrasonic Auger for Narrow-Gauge Borehole Drilling
}

\author{
David Firstbrook \\ School of Engineering \\ University of Glasgow \\ Glasgow, United Kingdom \\ david.firstbrook@glasgow.ac.uk \\ Thaleia Flessa \\ School of Engineering \\ University of Glasgow \\ Glasgow, United Kingdom
}

\author{
Kevin Worrall \\ School of Engineering \\ University of Glasgow \\ Glasgow, United Kingdom \\ Euan McGookin \\ School of Engineering \\ University of Glasgow \\ Glasgow, United Kingdom
}

\author{
Patrick Harkness \\ School of Engineering \\ University of Glasgow \\ Glasgow, United Kingdom
}

\author{
Douglas Thomson \\ School of Engineering \\ University of Glasgow \\ Glasgow, United Kingdom
}

\begin{abstract}
An ultrasonic auger was designed, manufactured, and tested in glass microspheres to investigate force and torque reducing effects upon application of highpowered ultrasonic vibration. It was found that whilst vibration had no effect on overhead force during rotary augering, the torque was reduced by up to $30 \%$, depending on amplitude of vibration.
\end{abstract}

Keywords-drilling, auger, high-power ultrasonics, borehole, granular material, sub-surface.

\section{INTRODUCTION}

The "roBot for Autonomous unDerGround trenchless opERations, mapping and navigation" (BADGER) is a project to design, develop, and build an autonomous underground robotic system to drill, manoeuvre, map, and navigate the subsurface [1]. This Horizon 2020 project is also equipped with construction capabilities in order to create a network of bores and tunnels. BADGER will be connected to a ground station only by a wireline, resulting in a single key-hole on the surface for installation, minimising any disruption to the surface.

An issue with the wireline approach is that the weighton-bit (WOB), as well as the torque reaction required for regular drilling must be provided downhole, rather than by the usual large supporting drilling rig. The BADGER device solves this issue by using inflatable air clamps to grip the borehole. As part of a plan to miniaturise the BADGER device for use in narrow-gauge drilling, integration of ultrasonic vibration to the drill head has been considered, due to its force reduction properties [2]. A reduction in the WOB and torque requirements will allow a smaller device to have more manoeuvrability, with less overall mass and complexity of supporting architecture.

This work will discuss the experiments taken to investigate the effects of using an ultrasonic auger with a granular material substrate, as well as the characterisation of the auger. The results and implications will be discussed at the end, with an overview of what direction the technology could go in.

\section{CHARACTERISING THE UltRASONIC AUGERS}

\section{A. Design of the augers}

A defining characteristic of an auger is the spiral flute that is designed to take spoil away from the drilling site by transferring rotational movement to linear. Two common methods of achieving this are the centrifugal effect, and the screw method [3].

The centrifugal effect is heavily dependent on rotations per minute (RPM) of the auger, balancing centrifugal with frictional force in order to ensure spoil is evacuated properly and efficiently. The screw method on the other hand is independent of rotation speed, relying on pure friction like in an ice-screw, or some method of containing the material, like in a water-screw. The auger used for the BADGER project operates similarly to the screw, and is not dependant on rotation speed.

Spoil removal in the larger BADGER device is achieved via spoil falling through holes in the front of the drilling face, where an internal vacuum transports it to surface level. For the narrow bore drilling concept discussed in this work, spoil from in front of the drill will be displaced either by compressing it to the sides of the device, or ultimately conveyed behind the drilling assembly. Either of these two methods will allow deep mole-like drilling to occur, as a long auger assembly up to the surface is not required.

The design of an ultrasonic auger requires compromise to achieve a shape that can auger spoil effectively, but also be able to operate within the constraints given by ultrasonic resonance. The ultrasonic signal was provided by a Sonic Systems P100 that was able to automatically track and supply any shift in resonant frequency whilst under load. The range it is able to handle is within $19.5 \mathrm{kHz}-20.5 \mathrm{kHz}$, and so the auger was designed to show a strong mode of vibration at $20 \mathrm{kHz}$ in order to allow for shifting during loading.

Previous work investigating ultrasonic vibration and granular material utilised the longitudinal mode for a simple penetrator, and showed a large decrease in both total consumed power as well as required overhead force [2]. Continuing this work, the longitudinal mode was also chosen for use with this auger. To keep symmetry, the auger incorporated a double-helix scroll, and can be seen in Fig. 1.

For the best combination of strength, manufacturability, and acoustic properties, the material chosen was stainless steel 316 .

\section{B. Finite Element Analysis (FEA)}

Due to the complicated shape, the auger was first designed in SolidWorks, then exported to Abaqus to conduct the Finite Element Analysis (FEA) of vibration. The longitudinal mode and $20 \mathrm{kHz}$ resonance requirements limit 


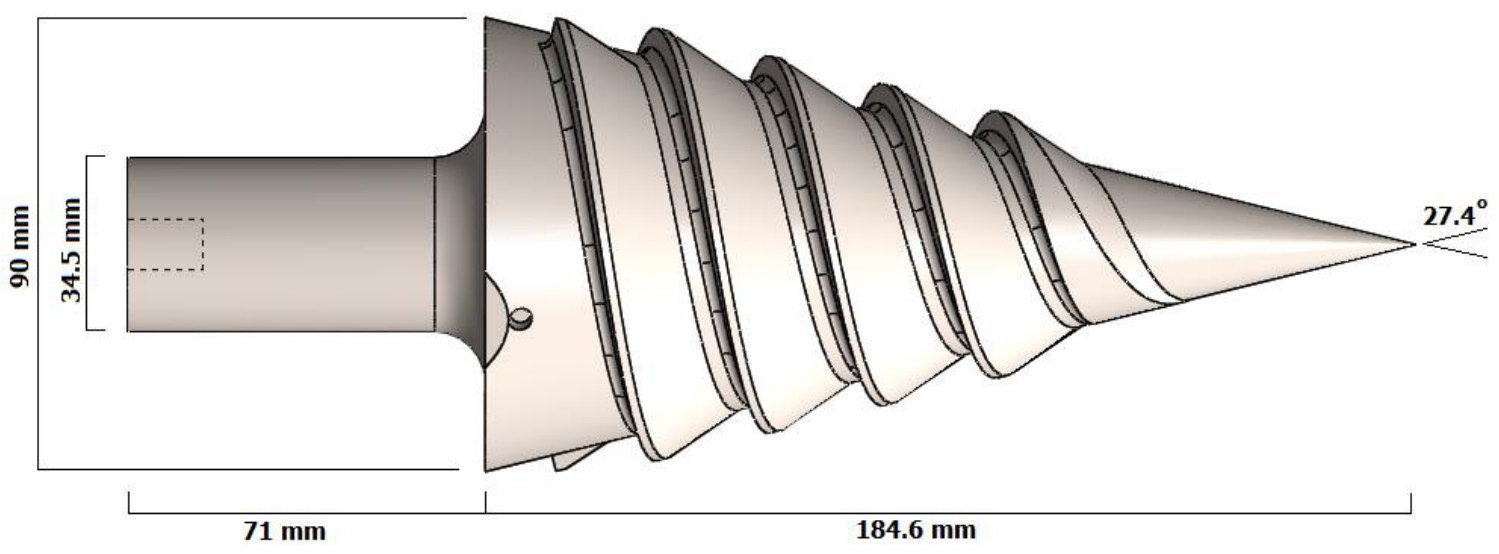

Fig. 1. Shape and dimensions of the stainless steel auger used in these experiments.

the overall length of the auger. Due to this, the second longitudinal mode (L2) was chosen over L1 for the extra length afforded. A recursive design process of analysis and re-design was undertaken in order to achieve resonance near $20 \mathrm{kHz}$. Following common practise, the actual design sought a slightly lower resonance point, to allow for material to be removed from the base for fine-tuning.

The auger was required to be attached to the front of an existing drill stem, which set the width of the auger-cone. Additionally, the gap between the auger and the drill stem needed to be sealed against spoil ingress so that the piezoelements would not be damaged. A polytetrafluroethene (PTFE) ring placed at the base of the auger provided an adequate seal against dirt, and the auger was designed to have the vibrational nodal plane as close to this point as possible, to reduce any heating or energy losses in the system due to contact rubbing.

After several iterations, a balance of dimensions was reached that satisfied all criteria. The auger displayed the L2 at $19.8 \mathrm{kHz}$, with the next nearest modes at 17.7 for the B2 mode, and $20.4 \mathrm{kHz}$ for the $\mathrm{B} 3$ mode.

The bending modes that fall within the trackable range of $19.5 \mathrm{kHz}-20.5 \mathrm{kHz}$ could potentially cause issues with the smooth operation of the auger. The physical vibrational modes were analysed after manufacture to see what the magnitude of these unwanted modes would be, and if they could be a significant problem. The theoretical gain of the auger, the ratio of tip to base amplitude, was 1.51

\section{Experimental Modal Analysis (EMA)}

The Experimental Modal Analysis (EMA) was conducted using a white noise input signal to the transducer, with the resultant vibration recorded by a Polytec CLV 3D laser vibrometer, and the results visualised in ME'scope. The frequency response between $12 \mathrm{kHz}$ and $28 \mathrm{kHz}$ can be seen in Fig. 2.

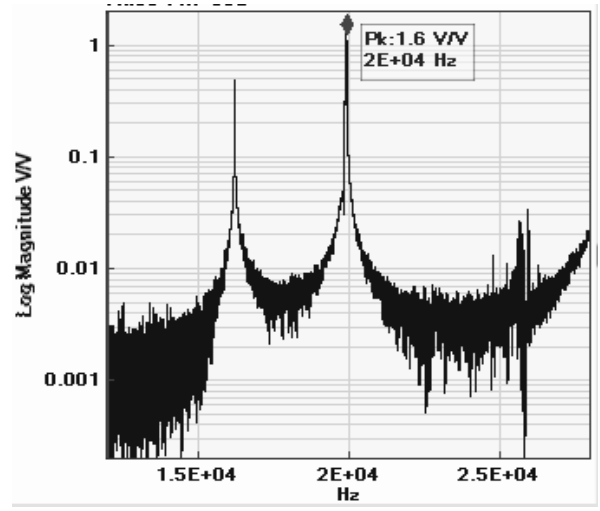

Fig. 2. Frequency response of the steel auger.
The L2 mode showed peak resonance at $19.925 \mathrm{kHz}$. This was slightly higher than the FEA predicted, however it is well within reason, and falls within the tracking range of the Sonic Systems signal supply. No other modes were present within this range, with the next closest resonant mode located at $16.3 \mathrm{kHz}$; well outside of concern.

Using these experimental results, the gain of the auger was determined to be 1.53 ; slightly higher than predicted.

\section{EXPERIMENTAL OVERVIEW}

\section{A. Granular material}

Glass micro-spheres were chosen due to their wellcharacterised properties and uniform spherical shape. The particle size distribution ranged from $150 \mu \mathrm{m}$ to $250 \mu \mathrm{m}$ to closely match the distribution of fine-sand [4] used for similar ultrasonics and granular material experiments [2].

The testing container was filled with the microspheres, and levelled off to measure the height of the material. This was used to calculate the volume of sand, and combined with the total weight was used to calculate the total bulk density. With a total mass of $276.54 \mathrm{~kg}$ of material, and a volume of $0.186 \mathrm{~m}^{3}$, the glass microspheres in these tests had a bulk density of $1,487 \mathrm{~kg} / \mathrm{m}^{3}$. The height and the diameter of material, $73 \mathrm{~cm}$ and $57 \mathrm{~cm}$ respectively, were chosen to reduce the levels of boundary effects that can be seen in granular material experiments [5]. It is impossible to completely get rid of boundary effects in any container, and a compromise must be reached between the size of container and practicalities of testing. The same container was used and the bulk density was constant across all tests, allowing a consistent granular fabric for each drilling run.

Previous work has shown that repeated penetration into the same sample of granular material will result in progressively higher force profiles [6]. This is due to the penetrator compacting the sand in the immediate region, effectively leaving a 'wake' of densified sand behind. Due to the large mass of material used in these tests, it was not possible to move the material to another container to reset the granular fabric.

Instead, it was recirculated by hand using a large mixer ensuring the whole volume was recirculated, thereby breaking apart any formation of densified sand. The consistency of this method is largely based on the operator's abilities, so to improve accuracy the same operator reset all samples of material. Additionally, the rotational tests were 
repeated five times and averaged, and the static penetration tests were repeated three times and averaged.

\section{B. Experimental procedure}

All drilling runs used a constant feed rate of $8 \mathrm{~mm} / \mathrm{s}$ to a final depth of $300 \mathrm{~mm}$. Base ultrasonic excitation amplitudes, set by the Sonic Systems supply pack, were chosen to be $1,2,5$, and $10 \mu \mathrm{m}$ to incorporate a wide range of amplitudes, in addition to a non-ultrasonic run which will hereafter be referred to as $0 \mu \mathrm{m}$. The physical amplitude at the tip is thus the gain multiplied by the base values, however for comparison purposes the base excitation value is used for these results.

Running the auger motor at the optimum rotations per minute (RPM) resulted in a final auger rotation speed of 14.7 RPM, after all the gear ratios in the system were accounted for. A perfect screw theoretically requires no overhead force in order to progress through a material, relying solely on the rotation and spiral shape. Thus to isolate and investigate the effect that rotation has on the progression and overhead force requirements the experiments were repeated at 0 RPM, referred to as the non-rotary tests.

Torque of the system was provided by the Maxon motor software. This measures the torque experienced by the motor. To calculate the torque experienced by the auger, this value must be converted by multiplying it by the compound gear ratios, as well as the transmission efficiency of the motor $(76 \%)$ and of the gearboxes $(60 \%)$ [7]. The torque shown in the results is the torque experienced by the auger. The experimental rig is shown in Fig. 3.

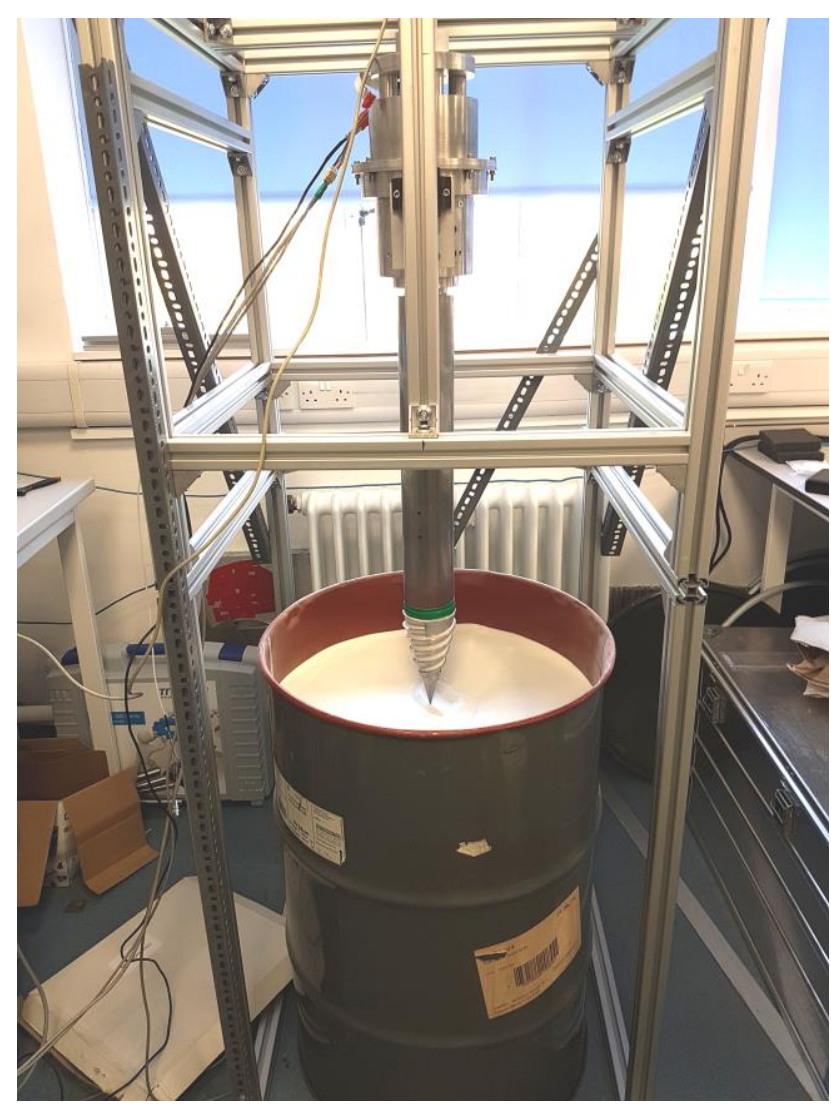

Fig. 3. The experimental ultrasonic augering rig. The auger motor, drill stem, and auger are connected to a rail, allowing linear movement.

\section{RESULTS AND ANALYSIS}

\section{A. Force effects}

The effects of ultrasonic vibration on overhead force can be seen in Fig. 4, showing the peak reacted force for both the rotary and non-rotary experiments. Force increases steadily with depth, with the maximum depth of $30 \mathrm{~cm}$ corresponding to the peak force.

It is clear that the non-rotary penetration requires significantly more force than in the rotary tests. Overhead force is reduced from roughly $600 \mathrm{~N}$ to $100 \mathrm{~N}$ in the nonultrasonic case, whereas it is reduced to roughly $350 \mathrm{~N}$ at maximum amplitude for no rotation. This suggests that the cork-screw effect caused by rotation has a much larger impact on force reduction than ultrasonic vibration does. This would explain why increasing vibration appears to have no effect on reducing overhead in rotary augering; the force has already been reduced by more than ultrasonics can provide.

\section{B. Torque effects}

The effects of ultrasonic vibration on torque can be seen in Fig. 5. Similar to force, the torque steadily increases with increasing depth, reaching a peak at the maximum depth of $30 \mathrm{~cm}$.

Here, a clear decrease in the peak torque with increasing ultrasonic amplitude is evident, decreasing from $120 \mathrm{Nm}$ to $85 \mathrm{Nm}$ at $10 \mu \mathrm{m}$; a $30 \%$ overall decrease. The ultrasonic vibration causes granular fluidisation in the immediate surrounding of the auger, reducing the surface friction.

It is important to note the power consumption from loading the ultrasonic transducer in this way. The power consumption at the maximum depth was measured to be roughly $10,25,120$, and $300 \mathrm{~W}$ for $1,2,5$, and $10 \mu \mathrm{m}$ respectively, for the augering tests. For terrestrial applications such as the BADGER device, this level of power consumption is manageable, as the device is tethered to a ground support unit where power supply in not a constriction.

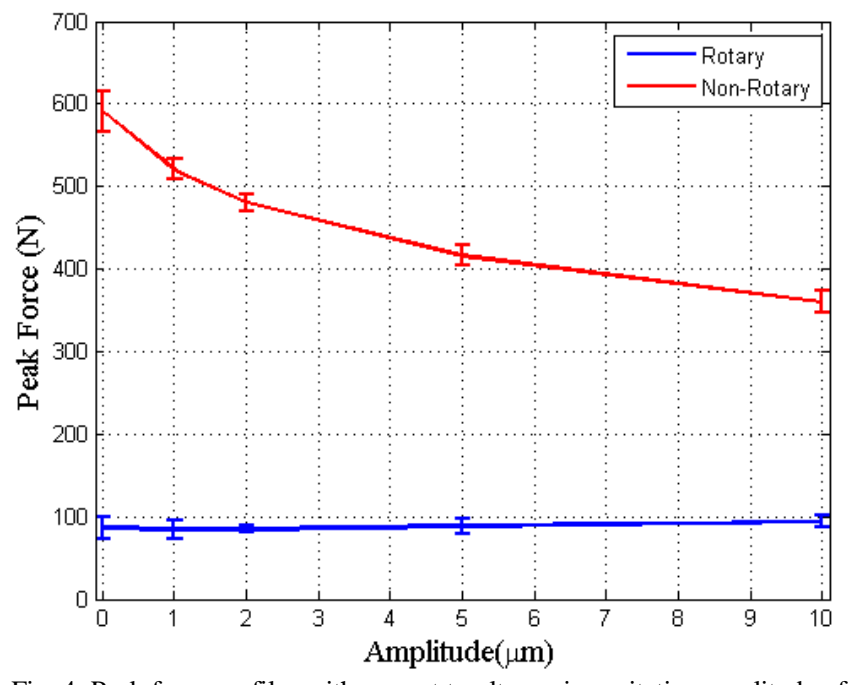

Fig. 4. Peak force profiles with respect to ultrasonic excitation amplitude of rotary and non-rotary augering. Rotary data points were repeated five times, and non-rotary repeated three times. Error bars show 1 standard deviation from the mean. 


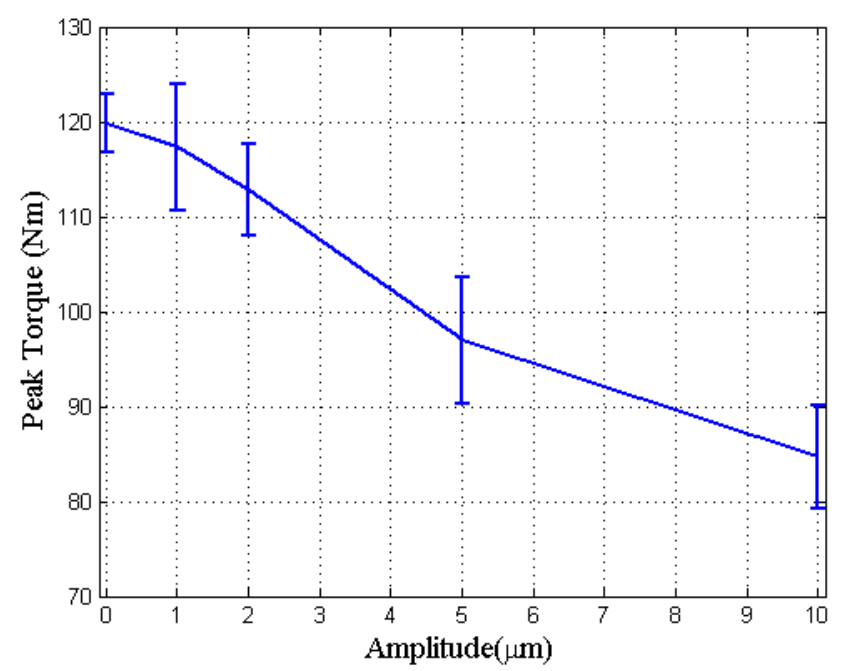

Fig. 5. Peak torque profile with respect to ultrasonic excitation amplitude of rotary augering. Rotary data points were repeated five times. Error bars show 1 standard deviation from the mean.

\section{CONCLUSION}

The force and torque reaction requirements for an ultrasonic auger were experimentally examined. It was found that whilst ultrasonic vibration might assist in reducing overhead force for static penetration, it appears to not alter force if augering is taking place. However, ultrasonic vibration does reduce the torque reaction requirements, reducing by up to $30 \%$ in some cases.

BADGER will provide torque reaction via an inflatable clamp, and efforts to miniaturise BADGER face difficulties with being able to supply enough torque for a small size. Employing ultrasonic vibration on the augering tip has shown promise in reducing torque to manageable levels in this case, and is an attractive solution for any small-scale drill or auger device.

[1] http://badger-robotics.eu/ (accessed $5^{\text {th }}$ October 2018)

[2] D. Firstbrook, K. Worrall, R. Timoney, F. Suñol, Y. Gao, and P. Harkness, "An Experimental Study of Ultrasonic Vibration and the Penetration of Granular Material," Proc. R. Soc. A, vol. 473, no. 2198, pp. 1-16, 2017.

[3] K. Zacny and G. Cooper, "Methods for cuttings removal from holes drilled on Mars," Int. J. Mars Sci. Explor., vol. 3, pp. 42-56, 2007.

[4] C. K. Wentworth, "A Scale of Grade and Class Terms for Clastic Sediments," J. Geol., vol. 30, no. 5, pp. 377-392, 1922.

[5] M. D. Bolton et al., "Centrifuge cone penetration tests in sand," Géotechnique, vol. 49, no. 4, pp. 543-552, 1999.

[6] K. Seiferlin et al., "Simulating Martian regolith in the laboratory," Planet. Space Sci., vol. 56, no. 15, pp. 2009-2025, 2008.

[7] www.maxonmotor.co.uk (accesed $5^{\text {th }}$ October 2018. see motor part no. 283858 and gear part no. 166951) 